\title{
Erratum to: Elevated growth differentiation factor 15 expression predicts poor prognosis in epithelial ovarian cancer patients
}

\author{
Ying Zhang ${ }^{1,2}$ - Wei Hua ${ }^{5}$ - Li-chun $\mathrm{Niu}^{2}$ • Shi-mei $\mathrm{Li}^{2}$ • Ying-mei Wang ${ }^{3}$ • \\ Lei Shang ${ }^{4}$ - Cun Zhang ${ }^{1}$. Wei-na $\mathrm{Li}^{1} \cdot$ Rui Wang ${ }^{4} \cdot$ Bi-liang Chen ${ }^{5}$. \\ Xiao-yan Xin ${ }^{5} \cdot$ Ying-qi Zhang ${ }^{1} \cdot$ Jian Wang $^{5}$
}

Published online: 12 March 2016

(C) International Society of Oncology and BioMarkers (ISOBM) 2016

\section{Erratum to: Tumor Biol}

DOI 10.1007/s13277-015-4699-x

The original version of this article unfortunately contained an error in the author group section.

The superscript of co-author Wei Hua should have been 5 not

3 . The corrected author group is shown above.

The online version of the original article can be found at http://dx.doi.org/ 10.1007/s13277-015-4699-x.

Ying-qi Zhang

zhangyingqi710032@163.com

$凶$ Jian Wang

wangiian710032@163.com

1 The State Key Laboratory of Cancer Biology, Biotechnology Center, School of Pharmacy, The Fourth Military Medical University, 17 Changle West Road, 710032 Xi'an, Shaanxi, China

2 Department of Gynecology and Obstetrics, The People's Liberation Army 323 Hospital, Xi'an, Shaanxi 710045, China

3 State Key Laboratory of Tumor Biology, Department of Pathology, Xijing Hospital, The Fourth Military Medical University, Xi'an, Shaanxi 710033, China

4 Department of Health Service, School of Public Health, Fourth Military Medical University, Xi'an, Shaanxi 710033, China

5 Department of Gynecology and Obstetrics, Xijing Hospital, Fourth Military Medical University, 169 Changle West Road,

710033 Xi'an, Shaanxi, China 